\title{
Numerical Study on Unstable Perturbation of Intrinsic Localized Modes in Graphene*
}

\author{
Yusuke DOI ${ }^{* *}$ and Akihiro NAKATANI ${ }^{* *}$ \\ ** Department of Adaptive Machine Systems, Graduate School of Engineering, Osaka University \\ 2-1 Yamadaoka, Suita, Osaka 565-0871, Japan \\ E-mail: doi@ams.eng.osaka-u.ac.jp
}

\begin{abstract}
A nonlinear vibration mode, referred to as the intrinsic localized mode (ILM), in graphene is investigated based on precise numerical solutions obtained by the iteration method coupled with molecular dynamics simulations. We obtain ILMs for which the frequency is greater than the maximum frequency of the phonon bands of graphene. The amplitude and structure of ILMs indicate that these ILMs are simply vibration modes due to the nonlinearity of the system. Moreover stability analysis of ILMs in graphene is performed numerically by solving the eigenvalue problem of the monodromy matrix based on Floquet theory for periodic solutions. In all cases, the ILM was found to have unstable perturbation modes. The growth rate of the unstable perturbation modes exhibits complex change behavior as the period of ILMs changes. The structure and growth rate of the unstable perturbation modes can be classified into two types. The most unstable perturbation mode is also localized and provides cooperative motions of neighbor atoms of the ILM.
\end{abstract}

Key words : Intrinsic Localized Mode, Molecular Dynamics, Graphene, Instability

\section{Introduction}

The nonlinear lattice is a useful model for describing nonlinear vibration phenomena in discrete systems, such as crystals. Nonlinear dynamics occur in the system when largedisplacement or large-amplitude vibration are excited in the system, i.e., large deformation or high temperature. In the nonlinear region, various interesting dynamics occur in the system.

Intrinsic localized modes (ILMs) or discrete breathers (DBs) have attracted a great deal of interest in the field of nonlinear physics ${ }^{(1)(2)}$. Intrinsic localized modes are space-localized and time-periodic vibration modes that are excited in anharmonic lattice models. In lattice systems, possible frequency regions of vibration form phonon bands in small-amplitude approximation due to discrete transform invariance of the systems. In anharmonic lattices, it is possible to excite anharmonic vibrations with a frequency that is not in the phonon band. These vibration modes do not lose their energy because they do not resonate with the phonon modes. Therefore, long-life vibration modes with frequencies out of the phonon band are possible in the anharmonic lattices. These vibration modes are referred to as ILMs.

Intrinsic localized modes have been observed in various experiments involving micromechanical systems ${ }^{(3)}$, macroscopic structures ${ }^{(4)}$, and nonlinear waveguide arrays ${ }^{(5)}$. Atomicscale crystal structure can also be regarded as a nonlinear lattice because atoms are discrete elements and interactions between atoms can be described by nonlinear functions. Experimental evidence of the excitation of the ILM in materials has been reported ${ }^{(6),(7)}$.

Numerical studies on the ILM in materials have been also performed. Marín el al. ${ }^{(8)}$ investigated the excitation of mobile ILMs in model crystals in which atoms interact with Lenard-Jones potentials in order to explain the mechanism of microtrack formation in mica crystals. Yamayose has reported the spontaneous excitations of the ILM in graphene based

${ }^{*}$ Received 30 July, 2011 (No. 11-0486) [DOI: 10.1299/jmmp.6.71]

Copyright (c) 2012 by JSME 
on the modulational instability of zone boundary modes ${ }^{(9)}$. Excitation of the ILM in carbon nanotubes has also been reported by Kinoshita ${ }^{(10)}$.

Since the ILM is a high-energy localization in a narrow region, it has been pointed out that the ILM may play an important role in the mechanical behavior of materials. Cuevas investigated the interaction between an ILM and vacancies in a one-dimensional lattice based on the Morse potential ${ }^{(11),(12)}$. In more realistic systems, Shimada recently reported the StoneWales transformation due to the ILM in carbon nanotubes ${ }^{(13)}$ in MD simulations.

In order to better understand the results of the MD simulation of the ILM in materials, it is necessary to investigate the basic properties of the ILM from a theoretical viewpoint. In the present study, we investigate the structure and stability of ILMs in graphene using precise numerical solutions. These solutions are calculated by a numerical method based on the iteration method coupled with MD simulations. Linear stability is also investigated numerically based on Floquet theory for time varying linear differential equations by regarding the dynamic atoms in crystals as a dynamic system.

The remainder of the present paper is organized as follows. A model and numerical method for obtaining the precise numerical solution of ILMs are presented in Section 2. The numerical method of linear stability analysis of the ILM is presented along with a brief review of Floquet theory in Section 3. In Section 4, numerical results and discussions are presented, and conclusions are presented in Section 5.

\section{Models and numerical method}

Figure 1 shows the calculation model. We consider a two-dimensional graphene sheet. The unit cell is aligned five cells along the $x$-direction and nine cells along the $y$-direction. The total number of atoms in the system is 180 . Each atom moves only in the $x$ - and $y$ directions. A periodic boundary condition is assumed in the $x$-direction, and a fixed boundary condition is assumed in the $y$-direction. Tensile or compressive strain is introduced to the system by displacing the fixed boundary. In this case, we process the numerical simulations after relaxation of the system. For describing the interaction between atoms, we consider the heuristic model potential proposed by Brenner ${ }^{(14)}$.

$$
H=\sum_{i=1}^{N} \sum_{\alpha} \frac{\left(p_{i}^{\alpha}\right)^{2}}{2 m}+\frac{1}{2} \sum_{i}^{N} \sum_{j \neq i}^{N} \sum_{k \neq i, j}^{N} \Phi_{i, j, k}\left(r_{i j}, r_{i k}, \theta_{i j k}\right)
$$

where $i$ is an index of atoms, $\alpha$ is an index of dimension, $m$ is the mass of a carbon atom, $p_{i}$ is the momentum of the $i$-th atom, $r_{i j}=q_{j}-q_{i}$ is the relative displacement of two atoms, $q_{i}$ is the position of the $i$-th atom, and $\theta_{i j k}$ is the angle of bands $i-j$ and $i-k$. The equilibrium length between atoms is given as $0.145 \mathrm{~nm}$. We use the velocity Verlet method for numerical integration of Newton's equations of motion. The time step of the integration is set to $0.01 \mathrm{fs}$.

The ILM is a time-periodic solution. Therefore, the ILM takes a periodic orbit in the phase space. We find a precise numerical solution of the ILM by searching a periodic orbit with a frequency that is out of the phonon bands of graphene. Let $\mathbf{x}=\{\mathbf{q}, \mathbf{p}\}^{T}$ be state variables that indicate the state of the systems in the phase space. The temporal evolution of the state $\mathbf{x}$ is described by the equation of motion

$$
\dot{\mathbf{x}}=\mathbf{f}(\mathbf{x})
$$

where the function $\mathbf{f}$ is given by

$$
\mathbf{f}(\mathbf{x})=\left\{\frac{\partial H}{\partial \mathbf{p}},-\frac{\partial H}{\partial \mathbf{q}}\right\}^{T} .
$$

Formally integrating (2), we obtain

$$
\mathbf{x}(t)=\int_{t_{0}}^{t} \mathbf{f}(\mathbf{x}(\tau)) d \tau+\mathbf{x}\left(t_{0}\right)
$$




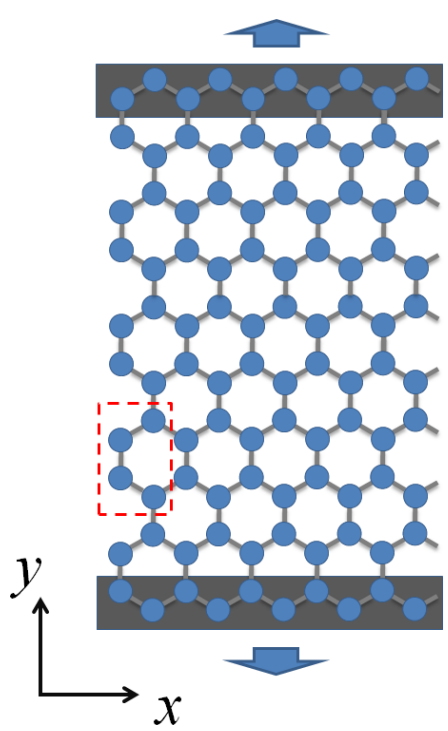

Fig. 1 Model. The region surrounded by the dashed line indicates a unit cell.

If we consider the temporal evolution of a period $T$ of the ILM, Eq. (4) becomes

$$
\mathbf{x}\left(t_{0}+T\right)=\int_{t_{0}}^{t_{0}+T} \mathbf{f}(\mathbf{x}(\tau)) d \tau+\mathbf{x}\left(t_{0}\right) .
$$

When $\mathbf{x}(\mathrm{t})$ describes the temporal evolution of the ILM with a period of $T, \mathbf{x}(t)$ forms a closed path in the phase space. Therefore, we obtain the following relation:

$$
\mathbf{x}\left(t_{0}+T\right)-\mathbf{x}\left(t_{0}\right)=\int_{t_{0}}^{t_{0}+T} \mathbf{f}(\mathbf{x}(\tau)) d \tau=\mathbf{0} .
$$

Finding an ILM with period $T$ is equivalent to solving Eq. (6).

We solve Eq. (6) for fixed $T$ using the conjugate gradient method. Integration of $f(\mathbf{x}(t))$ is performed by the MD method. In many particle systems, it is difficult to obtain a good initial guess of the periodic orbit. First, we solve Eq. (6) for small particle systems, in which a few atoms around the ILM are allowed to move. Once we find an appropriate solution for the small particle systems, we attempt to solve the equation for systems that have larger degrees of freedom, regarding the previous solution as the new initial guess.

\section{Linear stability analysis of the ILM}

The ILM is a time-periodic vibration mode. We can analyze the linear stability of the ILM by investigating the perturbation around the solution of the ILM. Let $\mathbf{x}=\mathbf{x}_{\mathrm{ILM}}(t)=$ $\left\{\mathbf{q}_{\mathrm{ILM}}(t), \mathbf{p}_{\mathrm{ILM}}(t)\right\}^{T}$ be a solution of the ILM. The variational equation of perturbation is obtained by substituting $\mathbf{q}(t)=\mathbf{q}_{\mathrm{ILM}}(t)+\xi(t)$ and $\mathbf{p}(t)=\mathbf{p}_{\mathrm{ILM}}(t)+\eta(t)$ into (2) and linearizing the result as follows:

$$
\begin{aligned}
& \dot{\xi}_{i}=\left.\sum_{j} \frac{\partial^{2} H}{\partial p_{i} \partial q_{j}}\right|_{\mathbf{x}=\mathbf{x}_{\mathrm{ILM}}(t)} \xi_{j}+\left.\sum_{j} \frac{\partial^{2} H}{\partial p_{i} \partial p_{j}}\right|_{\mathbf{x}=\mathbf{x}_{\mathrm{ILM}}(t)} \eta_{j}, \\
& \dot{\eta}_{i}=-\left.\sum_{j} \frac{\partial^{2} H}{\partial q_{i} \partial q_{j}}\right|_{\mathbf{x}=\mathbf{x}_{\mathrm{ILM}}(t)} \xi_{j}-\left.\sum_{j} \frac{\partial^{2} H}{\partial q_{i} \partial p_{j}}\right|_{\mathbf{x}=\mathbf{x}_{\mathrm{ILM}}(t)} \eta_{j}
\end{aligned}
$$

where $\xi$ and $\eta$ are the perturbations around position $\mathbf{q}$ and momentum $\mathbf{p}$, respectively. Coefficients of the variational equations, i.e., Eqs. (7) through (8), are found to be $T$-periodic, because the ILM solution $\mathbf{x}=\mathbf{x}_{\text {ILM }}(t)$ is $T$-periodic. Therefore, the variational equations can be also written as follows:

$$
\dot{\zeta}=A(t) \zeta, \quad A(t)=A(t+T)
$$

where $\zeta=\{\xi, \eta\}^{T}$. 
The ILM solution $\mathbf{x}=\mathbf{x}_{\mathrm{ILM}}(t)$ is a periodic solution, and the temporal evolution of the variational equations given in Eq. (9) has a linear relation:

$$
\zeta(t+T)=M(T) \zeta(t) .
$$

The matrix $M(T)$ is the monodromy matrix and includes information on the linear stability of the ILM. We can obtain the stability information by solving the eigenvalue problems of monodromy matrix $M(T)$.

$$
M(T) \zeta_{n}=\sigma_{n} \zeta_{n}
$$

The eigenvalues $\sigma_{n}$ of the monodromy matrix indicate the growth rate of the perturbation. In addition, the eigenvectors $\zeta_{n}=\left\{\xi^{n}, \eta^{n}\right\}^{T}$ indicate the structure of the corresponding perturbation modes. Arbitrary perturbation $\zeta$ can be decomposed into

$$
\zeta(t)=\sum_{n} a_{n}(t) \zeta_{n}
$$

Substituting Eq. (12) into Eq. (10) and applying Eq. (11), we obtain

$$
\zeta(t+T)=\sum_{n} a_{n}(t) \sigma_{n} \zeta_{n}(t)
$$

The perturbation modes of $\left|\sigma_{n}\right|>1$ grow after one period vibration of the ILM. Therefore, the ILM is unstable if eigenvalues $\sigma_{n}$ of the monodromy matrix of the ILM $M(T)$ exist such that $\left|\sigma_{n}\right|>1$. Equations (7) through (8) indicate that the variational equations are also Hamilton systems. Therefore, the monodromy matrix $M(T)$ is a symplectic matrix. In the case of a symplectic matrix, if $\sigma_{n}$ is an eigenvalue, then $1 / \sigma_{n}$ is also eigenvalue. This indicates that the ILM is linearly unstable if there exists an eigenvalue that is out of the unit circle on the complex plane.

In general, analytical expression of the monodromy matrix cannot be obtained. Instead, we can calculate the monodromy matrix numerically. Let the map $I(\mathbf{x})$ as the temporal evolution of $x$ during the period $T$,

$$
I(\mathbf{x})=\int_{\tau}^{\tau+T} \mathbf{x}(\tau) d \tau
$$

The monodromy matrix $M$ is obtained numerical as follows:

$$
M_{m n}=\frac{I_{n}\left(\mathbf{x}+\delta^{(\mathbf{m})}\right)-I_{n}(\mathbf{x})}{\left|\delta^{(m)}\right|}
$$

where $\delta^{(\mathbf{m})}(m=1,2, \ldots)$ is the orthogonal perturbation vectors ${ }^{(2)}$.

\section{Results and discussion}

\subsection{Structure of the ILM}

We obtain a series of numerical solutions of the ILM with a frequency greater than the maximum frequency of the linear phonon mode of graphene. Figure 2 shows the displacement pattern of the ILM in graphene. Two carbon atoms vibrate with a large amplitude. The direction of the vibration is along the bond of atoms. Atoms neighboring the two major atoms also vibrate, although only slightly. The amplitude of vibration decays rapidly as the distance from the center of the ILM increases.

The structure of ILMs obtained by the numerical method is similar to the structures observed in MD simulations ${ }^{(10)}$. However, the lifetime of ILMs is much greater than in the case of MD simulation. In the above-cited MD simulation, the ILM that is spontaneously excited in graphene vibrates at 26 cycles. In the present study, the ILM obtained by proposed numerical method vibrates at 500 cycles. This result indicates that the ILM can exist as nonlinear vibration modes in graphene. In MD simulations, thermal fluctuations also exist as small vibrations of atoms in the system. Thermal fluctuations act as perturbations on ILMs. Therefore, ILMs become unstable in the finite period of time. The ILMs obtained by the 


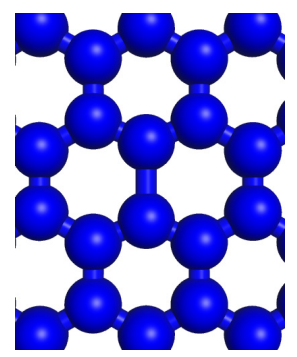

(a) $t=0 \mathrm{fs}$

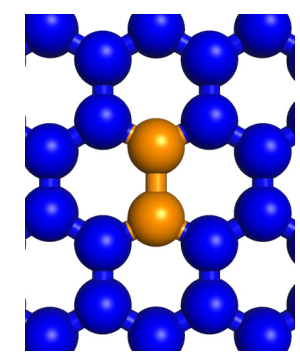

(b) $t=3 \mathrm{fs}$

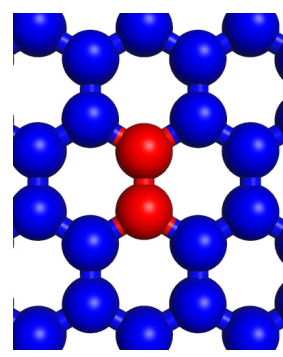

(c) $t=6 \mathrm{fs}$

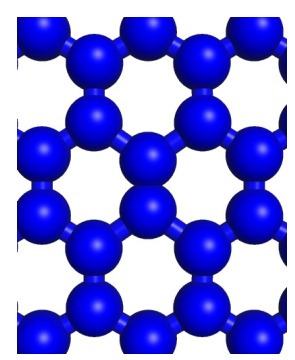

(d) $t=9.5 \mathrm{fs}$

Fig. 2 Snapshot of the vibration of the ILM in graphene with a period of $T=19 \mathrm{fs}$

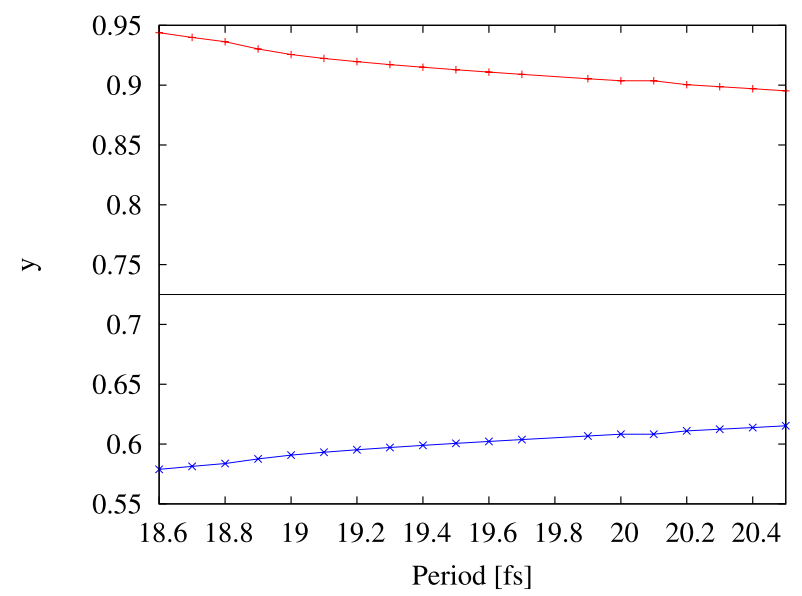

Fig. 3 Relation between the amplitude and period of the ILM. The red line is the maximum displacement in the expansion direction, and the blue line is the minimum displacement in the compression direction. The black line indicates the equilibrium position.

iteration method, on the other hand, do not include thermal fluctuations. Therefore, these ILM survive as long as the numerical errors of the integrations are sufficiently small.

The structure of the ILM is shown in detail. Figure 3 shows the relation between the period of the ILM and the maximum amplitude of the ILM. The amplitude of the ILM increases as the period of the ILM decreases. Therefore, the ILM vibrates due to positive nonlinearity, i.e., the curvature of the potential in terms of displacement is positive. In the case of Brenner potential, positive nonlinearity is dominant at the initial stage of displacement. The negative nonlinearity becomes dominant as the displacement increases. In region of negative nonlinearity, if the ILM exists, the amplitude of the ILM decreases as the frequency increases. In the calculation of the present study, the ILM is not obtained in the region in which the negative nonlinearity is dominant. This is because we search the solution of the ILM by increasing frequency of the ILM. When the amplitude of ILM reaches a critical point at which the positive nonlinearity and the negative nonlinearity exchange, convergence to a solution is difficult because the another solution with the same frequency exists in neighborhood.

Moreover, the amplitude of the ILM is asymmetric at the equilibrium position. In Fig. 3, the amplitude in the expansion direction is always greater than that in the compression direction. This asymmetry is due to the asymmetry of the interaction potential at the equilibrium position. At the same energy level, atoms can move a longer distant in the expansion direction than in compression direction. The particle energy in ILM is preserved during vibrations. Therefore, the amplitude of the ILM is observed to be asymmetric.

Note that the characteristics of the structure of the ILM discussed above, i.e., the dependence of the amplitude on frequency and asymmetric amplitude, are not observed in linear phonon modes or small-amplitude approximation. This means that the structure of the ILM 


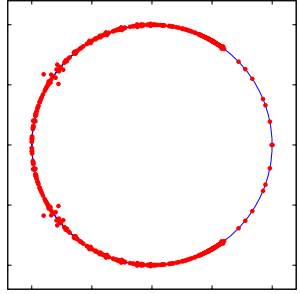

$T=18.6 \mathrm{fs}$

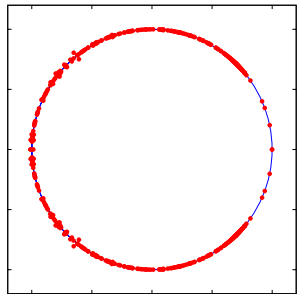

$T=19.5 \mathrm{fs}$

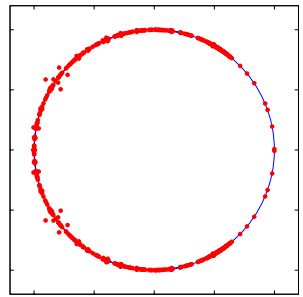

$T=18.8 \mathrm{fs}$

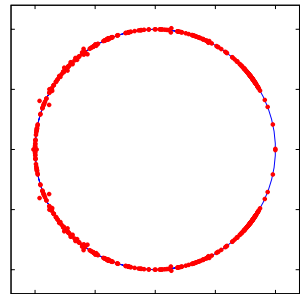

$T=20.0 \mathrm{fs}$

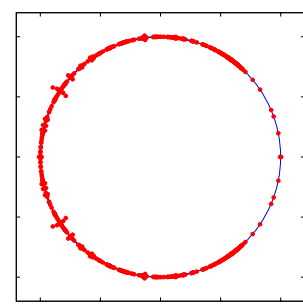

$T=19.0 \mathrm{fs}$

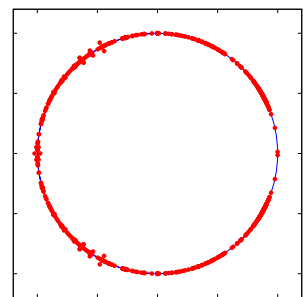

$T=20.5 \mathrm{fs}$

Fig. 4 Distribution of eigenvalues of the monodromy matrix in the complex plane. The blue line indicates the unit circle.

depends greatly on the characteristics of nonlinearity of the interaction between atoms, despite the universality of existence of the ILM in a wide range of nonlinear lattice systems.

\subsection{Linear stability of the ILM}

Figure 4 shows the distribution of eigenvalues of the monodromy matrix of the ILM with various periods in the complex plane. There are always eigenvalues outside the unit circle. Therefore, the ILM with a frequency that exceeds the maximum frequency of the phonon bands always has unstable perturbation modes. However, the position of eigenvalue that corresponds to the unstable perturbation modes moves as the period of the ILM changes. In the case of $T=20.5 \mathrm{fs}$, unstable eigenvalues are observed on the real axis. However, the unstable eigenvalues on the real axis disappear as the period $T$ becomes shorter. The unstable perturbation modes near $\theta= \pm 3 \pi / 4$ always appear, but vary as the period $T$ varies. These differences in the position of the unstable eigenvalues result in complex changes in the instability of the ILM.

Figure 5 shows the relation between the period $T$ of the ILM and the maximum growth rate of the unstable perturbation mode in the ILM. The growth rate exhibits complex changes as the period $T$ increases. However, the growth rate generally increases as the period of the ILM decreases. When the period of the ILM becomes short, the frequency of the ILM becomes high. This leads to an increase of the nonlinear effect of the interaction between atoms in the ILM, because the amplitude of the ILM becomes large. Therefore, the instability of the ILM increases as the number of nonlinear interactions between atoms increases. As mentioned above, we obtain the ILM with the frequency above the maximum frequency of the phonon bands. As the amplitude of the ILM increases, the atoms on the interaction potential approach the critical distance at which the positive nonlinearity and the negative nonlinearity of interaction are exchanged. Therefore, the ILM with a larger amplitude becomes more unstable.

Moreover, Fig. 5 indicates that there exist two regions in which the growth rate is higher and lower. The regions in which the growth rate is higher are 18.5-19.1 fs and 19.6-20.1 fs in terms of the period $T$ of the ILM. In these two regions, the characteristics of unstable perturbation modes are quite different. The growth rates of several unstable perturbation modes exhibit complex changes by the period of ILM. Moreover, the most unstable modes are swapped as a result of the strain of the graphene.

Here, we consider the variation in the growth rates due to the strain of the graphene. We 


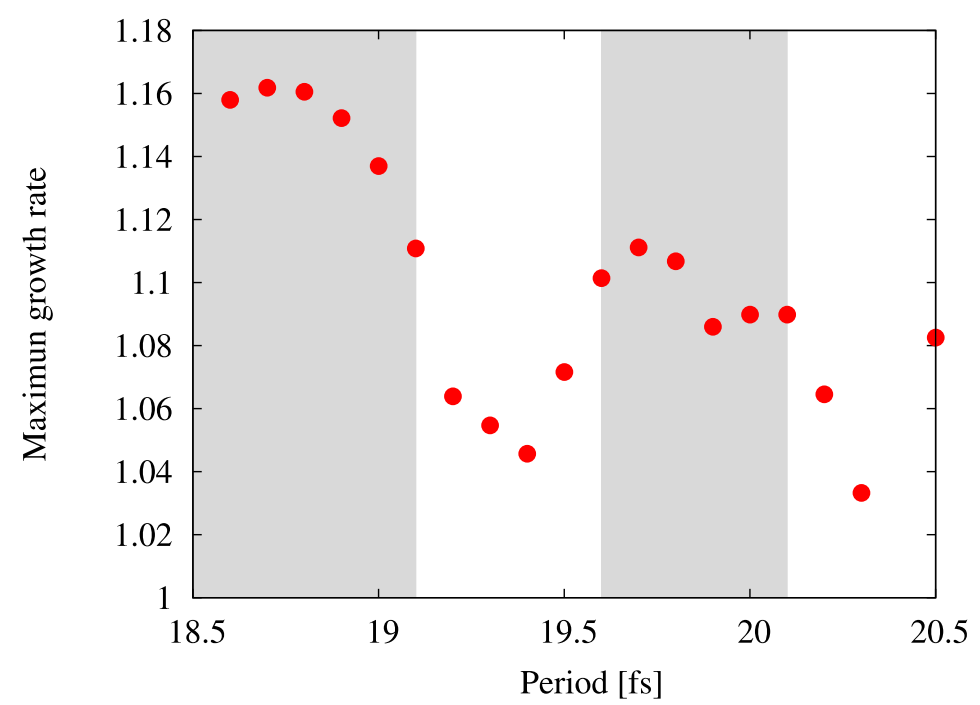

Fig. 5 Relation between the period of the ILM and the maximum growth rate of unstable perturbation mode. The gray bands indicate the region of higher growth rate.

consider the strain from $-10 \%$ (compressive) to $10 \%$ (tensile) by moving the fixed boundary of the system along the $y$-axis. Precise solutions of the ILMs are calculated after relaxation of the strained system. The strain is fixed in the numerical calculations conducted in order to search for a solution.

Figure 6(a) and (c) shows the relation between the strain and the growth rate of some unstable perturbation modes of the ILM with a period $T=19.0 \mathrm{fs}$ and $T=19.8 \mathrm{fs}$, respectively. This ILM corresponds to the region of the higher growth rate in Fig. 5. The most unstable perturbation modes change as the strain of the system increases. In the case of compressive strain, some peaks of the unstable perturbation modes are formed. In the case of tensile strain, on the other hand, exchange of the most unstable perturbation modes does not occur. The unstable perturbation mode with the highest growth rate is realized in tensile strain. Corresponding eigenvalues are located near $\theta=0.817 \pi$.

Figure 7 shows the structure of the unstable perturbation modes. In many cases, perturbation modes is localized structure in $y$-direction. In particular, in the case of most unstable perturbation mode, highly localized structure appears near the ILM. The shear motion of six atoms, including those in the ILM, is excited as a result of the instability of the ILM (righthand panel of Mode 4 in Fig. 7).

Figure 6(b) and (d) shows the relation between the strain and the growth rate of unstable perturbation modes of the ILM with a period of $T=19.5 \mathrm{fs}$ and $T=20.5 \mathrm{fs}$, respectively. This mode corresponds to the region of lower growth rate in Fig. 5. Compared to Fig. 6(a), the peaks of growth rates have different structures. Unlike the case in which $T=19.0 \mathrm{fs}$ and $19.8 \mathrm{fs}$, the most unstable growth rate is realized when compressive strain is introduced into the system. Corresponding eigenvalues are located near $\theta=0.716 \pi$. In the case of tensile strain, the unstable perturbation modes have relatively low growth rates.

Figure 8 shows the structure of the unstable perturbation modes. The unstable perturbation modes take localized structure as in the case of $T=19.0 \mathrm{fs}$. However, the structure of the perturbation modes differs from those in the case of $T=19.5 \mathrm{fs}$. In the case of the unstable perturbation mode with highest growth rate in compressive strain, the unstable perturbation mode has a symmetric structure of eight particles at the ILM (right-hand panel of Mode 2 in Fig. 8).

The above results indicate the essential difference between two regions in Fig. 5, i.e., the region of higher growth rate and the region of lower growth rate. We can see that the 


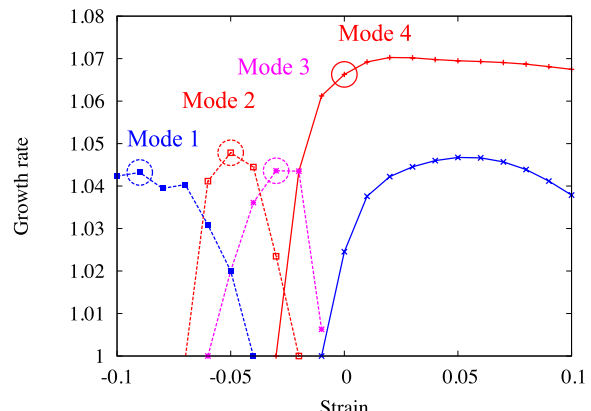

(a) $T=19.0 \mathrm{fs}$

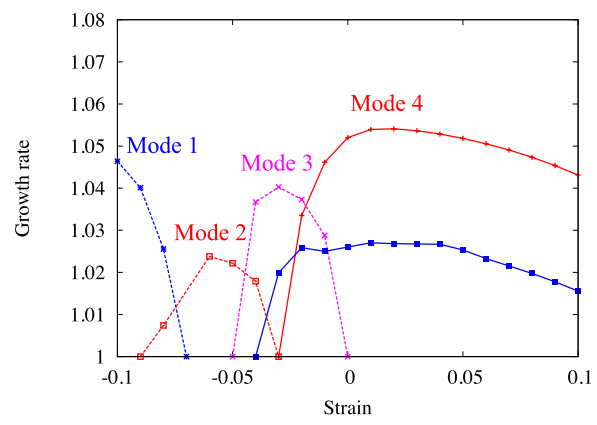

(c) $T=19.8 \mathrm{fs}$

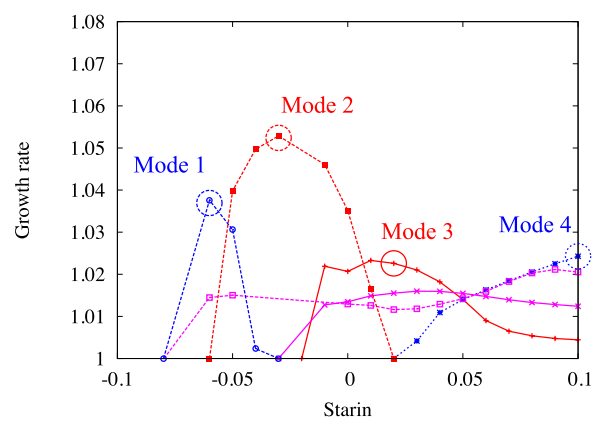

(b) $T=19.5 \mathrm{fs}$

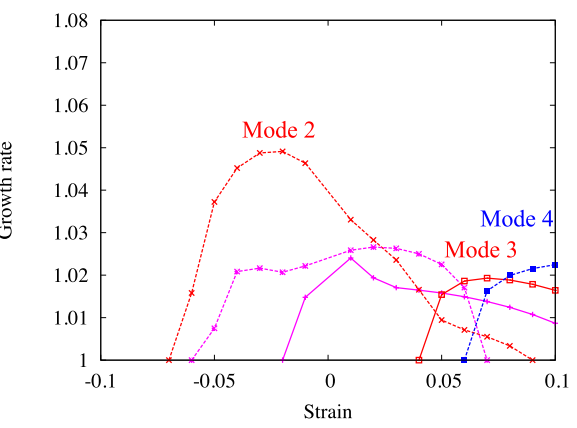

(d) $T=20.5 \mathrm{fs}$

Fig. 6 Relation between the strain of the system and the growth rate of major unstable perturbation modes: (a) and (c) the region of higher growth rate and (b) and (d) the region of lower growth rate in Fig. 5

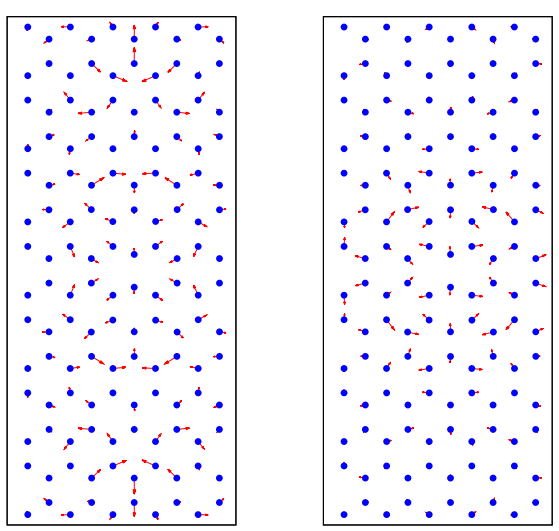

Mode 1

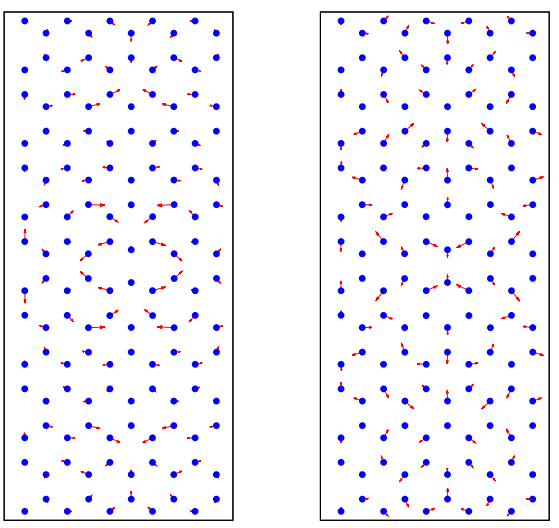

Mode 3

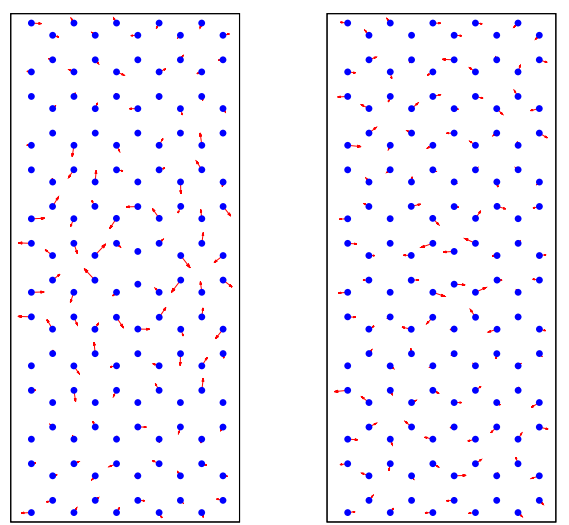

Mode 2

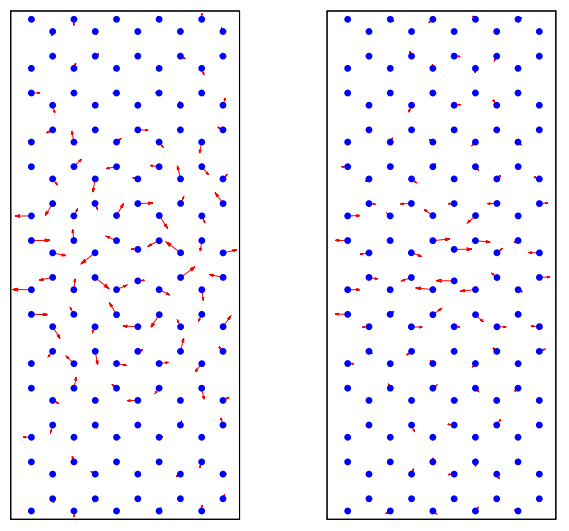

Mode 4

Fig. 7 Structure of major unstable perturbation modes for the ILM with $T=19.0 \mathrm{fs}$. Each mode corresponds to a labeled mode in Fig. 6(a). 


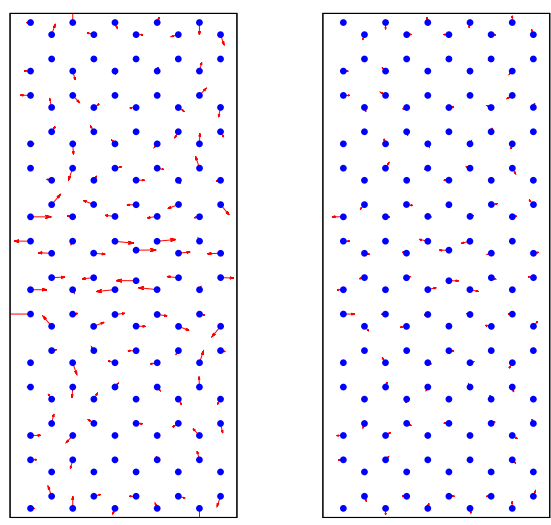

Mode 1

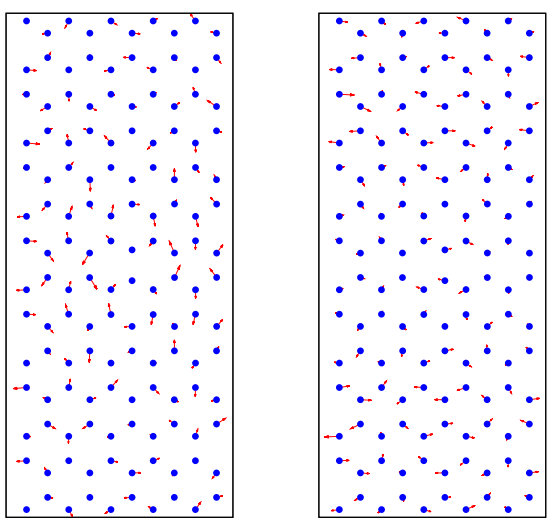

Mode 3

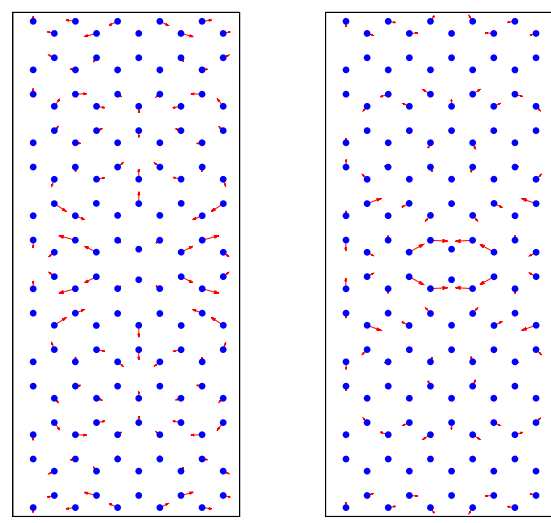

Mode 2

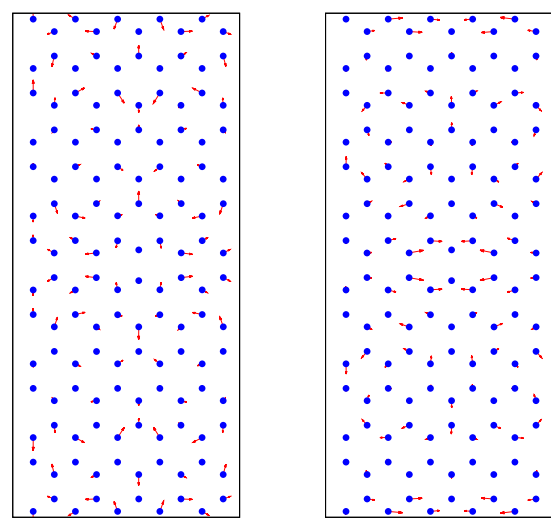

Mode 4

Fig. 8 Structure of major unstable perturbation modes for the ILM with $T=19.5 \mathrm{fs}$. Each mode corresponds to a labeled mode in Fig. 6(b).

same behavior of variation of growth rate in Fig. 6(a) and (c) for the region of higher growth rate, and in Fig. 6(b) and (d) for the region of lower growth rate. As to the structure of the perturbation mode, in both results, the most unstable perturbation modes localized near the particles in the ILM. This indicates that the instability of the ILM results in the cooperative motion of the surrounding atoms of the ILM. The structure of the perturbation modes, on the other hand, is quite different in the two results.

The growth rate is determined as the distance between the eigenvalues and the unit circles in the complex plane. Eigenvalues exit the unit circle when two eigenvalues on the unit circle that meet a certain condition collide. Eigenvalues move as the strain of the system varies. Therefore, the frequency of collision of two eigenvalues depends on the initial distribution of eigenvalues when no strain is introduced into the system. In Fig. 4, the band of eigenvalues is found to shrink as the period of the ILM decreases. A denser distribution of eigenvalue leads to frequent collision of eigenvalues. Therefore, unstable perturbation modes can frequently be produced in the case of a shorter period $T$ of the ILM, yielding more unstable perturbation modes. On the other hand, the mechanism responsible for the formation of two growth rate regions, as shown in Fig. 5 has not yet been clarified. This problem is left for future investigation.

The dynamics of crystals after instability is also interesting problem. In our preliminary calculations, the structure of the ILM deviated due to the existence of unstable perturbation modes. However, the ILM is not destroyed but rather is transformed into a quasi-periodic vibration mode, because we consider the Hamilton systems in our calculations. Investigation of the ILM in the system with a heat bath is required in order to obtain a better understanding of post-instability dynamics of ILMs. 


\section{Conclusion}

We investigated the basic properties of the ILM in graphene based on precise numerical solutions of ILMs. The structure of the ILM obtained by the iteration method coupled with MD simulation indicates the existence of some nonlinear intrinsic properties: large amplitude vibration, frequency dependence on amplitude, and asymmetric structure of amplitude. We also investigated the linear stability of the ILM. The ILM having a frequency greater than the maximum frequency of the phonon band was found to always be linearly unstable. The growth rate of the unstable perturbation modes exhibits complex variation in terms of the period of the ILM and the strain of graphene. We can classify the instability of the ILM into two types: higher-growth-rate instability and lower-growth-rate instability. The structure of the most unstable perturbation modes is also localized. These unstable perturbation modes lead to cooperative motion of the surrounding atoms of the ILM.

\section{Acknowledgement}

The present study was supported in part by a Grant-in-Aid for Young Scientists (B), No.22760066, from MEXT Japan.

\section{References}

( 1 ) Sievers, A.J. and Takeno, S., Phys. Rev. Lett., 61, pp.970-973, 1988.

( 2 ) Flach, S. and Gorbach, A.V., Phys. Rep., 467, pp.1-116, 2008.

( 3 ) Sato M. et al., Phys. Rev. Lett., 90, 044102, 2003.

( 4 ) Kimura, M and Hikihara, T, Chaos, 19, 013138,2009.

( 5 ) Eisenberg, H.S. et al., Phys. Rev. Lett., 81, pp.3383-3386, 1998.

( 6 ) Russell, F.M. and Eilbeck, J.C., Europhys. Lett., 78, 10004, 2007.

( 7 ) Manley, M.E. et al., Scientific Reports, Vol. 1, 4, 2011.

( 8 ) Marín, J.L., Eilbeck J.C. and Russell, F.M., Phys. Lett. A, 248, pp.225-229, 1998.

( 9 ) Yamayose, Y. et al., Europhys. Lett., 80, 40008, 2007.

(10) Kinoshita, Y. et al., Phy. Rev. B, 77, 024307, 2008.

(11) Cuevas, J. et al., Phys. Lett. A, 315, pp.364-371, 2003.

(12) Cuevas, J. et al., Physica D, 216, pp.115-120, 2006.

(13) Shimada, T., Shirasaki, D. and Kitamura, T., Phys. Rev. B, 81, 035401, 2010.

(14) Brenner, D.W., Phys. Rev. B, 42, pp.9458-9471, 1990. 